\title{
Technical note: Comparing calibration methods for determination of protein in goat milk by ultraviolet spectroscopy
}

\author{
E. O. Rukke, ${ }^{1}$ E. F. Olsen, T. Devold, G. Vegarud, and T. Isaksson \\ Department of Chemistry, Biotechnology and Food Science, Norwegian University of Life Sciences, 1432 Ås, Norway
}

\begin{abstract}
A rapid spectroscopic method to determine total protein in bovine and buffalo milk using UV spectra of guanidine-hydrochloride mixed milk has previously been reported and validated. The method was based on mixed calibration samples and univariate calibrations of fourth derivative (4D) spectra. In this study the same method was compared and tested for determination of total protein in goat milk. Calculations based on multivariate calibration (partial least squares regression) on full spectra of goat milk were used. The method was tested on 2 UV instruments. The comparison resulted in a significantly more robust (i.e., better) transferability between UV instruments for the partial least squares regression method on full spectra compared with previous univariate calibration of $4 \mathrm{D}$ spectra. Local (1 instrument) calibrations gave similar, significantly not different (chi-squared test) cross-validated prediction error results for the 2 methods. It can be concluded that there is no need for fourth derivation. Partial least squares regression on full spectra was equal or superior to using the $4 \mathrm{D}$ spectra.
\end{abstract}

Key words: milk protein, quantitative determination, ultraviolet spectroscopy, multivariate calibration

Milk has a short shelf life. Because the quality of milk-based products generally depends on processing the milk as fresh as possible, rapid determination of the content and quality regarding proteins and other constituents is of great importance. Changes in dairy product purchasing patterns have increased the value of milk protein over the last decades (Barbano and Lynch, 1992). The dairy industry has found it useful to include protein in milk pricing. These trends seem to continue because of low-fat products, nutritional recommendations, and so on.

At present, a large variety of spectrophotometric protein estimation methods exist for milk, such as infrared spectroscopy and methods that use other wavelengths

Received October 20, 2009.

Accepted March 29, 2010.

${ }^{1}$ Corresponding author: elling-olav.rukke@umb.no of the electromagnetic spectrum. In the dairy industry, the IR Milco Scan measurement system (Foss Electric, Hillerød, Denmark) is well known worldwide for analysis of protein in milk. But for smaller laboratories that are not so well equipped or in cases where just a few samples are analyzed, it is useful to have a simple, rapid method available that is accurate and economical. The described method also avoids dye-protein complexes that usually have to be removed by centrifugation or filtration before spectroscopic measurement (Sherbon, 1978).

Lüthi-Peng and Puhan (1999) and Miralles et al. (2000a,b) reported UV measurements on milk from cows and buffalo. This study focuses on goat milk. Goat milk has a long tradition in the Norwegian diet and is used as a supplement in the diet of humans who are allergic to cow milk (Liland et al., 2009). The measurements have been carried out in the wavelength range of 250 to $320 \mathrm{~nm}$, corresponding to absorbance of the aromatic AA residues Trp, Tyr, and Phe in the milk proteins. The AA Trp and Tyr are the main contributors to the protein absorption spectrum. There is about 4 times more Tyr (about $0.169 \mathrm{~g} / 100 \mathrm{~g}$ of milk) than Trp (Posati and Orr, 1976) in both cow and goat milk. LüthiPeng and Puhan (1999) concluded that the amplitude of the peak $283.5 \mathrm{~nm}$ should be used to quantify the total protein content in milk because both Trp and Tyr residues contribute to absorption at this wavelength.

The aim with the present study was to test, compare, and further develop the method of Lüthi-Peng and Puhan (1999) with alterations including 1) use of goat milk, 2) multivariate calibration, and 3) $2 \mathrm{UV}$ instruments.

In this study we used milk from 82 individual goats: 50 goats from the herd at The University of Life Sciences at Ås (Norway) and 32 goats from a farm at the northwest coast of Norway, Røyrhus (in Møre and Romsdal). The samples $(40 \mathrm{~mL})$ were all preserved with 2-bromo-2-nitropane-1,3-diol (Bronopol, D \& F Control Systems Inc., Norwood, MA) and cooled to $4^{\circ} \mathrm{C}$. The milk was skimmed at $4^{\circ} \mathrm{C}$ by centrifugation and separated into 2 fractions, skim milk and fat. The skim milk samples were divided in 2 parts, one for reference protein analysis and the other for spectroscopic analy- 
Table 1. Total milk protein, casein, and whey protein determinations (wt/wt \%) of 82 goat milk samples by Kjeldahl reference methods ${ }^{1}$

\begin{tabular}{lcccc}
\hline Constituent & Range & Average & $\mathrm{S}_{\text {Tot }}$ & $\mathrm{S}_{\text {Ref }}$ \\
\hline Total protein & $1.72-3.31$ & 2.64 & 0.32 & 0.028 \\
Casein & $0.79-2.17$ & 1.54 & 0.28 & 0.029 \\
Whey protein & $0.81-1.92$ & 1.09 & 0.19 & 0.007 \\
\hline
\end{tabular}

${ }^{1}$ Ordinary standard deviation $\left(\mathrm{S}_{\text {Tot }}\right)$ and standard error of the laboratory reference method $\left(\mathrm{S}_{\mathrm{Ref}}\right)$ are presented.

sis. Skim milk for UV analysis was stored in Eppendorf tubes at $-20^{\circ} \mathrm{C}$ until use.

Reference protein values for the calibration samples were determined by the Kjeldahl procedure (IDF, 1962, 1964) with modifications (Devold et al., 2000). Preparation of NPN fractions followed the procedure of Aschaffenburg and Drewry (1959). Milk (60 $\mu \mathrm{L})$ was added to $3 \mathrm{~mL}$ of guanidine acetate buffer $(6 \mathrm{M}$ guanidine- $\mathrm{HCl}$ in $0.1 \mathrm{M} \mathrm{Na}$ acetate buffer, $\mathrm{pH} 5.0$ ) and blended on a vortex mixer. Guanidine-HCl (SigmaAldrich, St. Louis, MO) acted as a denaturant of the proteins and at the same time reduced the turbidity of the milk by disintegration of the casein micelles. All mixing steps were gravimetrically determined (Mettler PM 480 Delta Range, Mettler Toledo AG, Greitensee, Switzerland) to develop the method for accurate quantitative protein determination based on the mass of protein. The content of protein throughout the work is expressed in weight/weight percentage. This weighing is a further development from the method of LüthiPeng and Puhan (1999). Duplicates of each sample were prepared. A 10-mm quartz cuvette was used, and UV spectra were recorded with the Guanidine- $\mathrm{HCl}$ buffer as reference on $2 \mathrm{UV}$ spectrophotometers at $20^{\circ} \mathrm{C}$. Average scanning time was $170 \mathrm{~s}$ scanning from 250 to $320 \mathrm{~nm}$ with intervals of $0.2 \mathrm{~nm}(0.5 \mathrm{~nm}$ in the paper of Lüthi-Peng and Puhan, 1999). The measurements were carried out by 2 instruments, a UV-1601 visible spectrophotometer (Shimadzu, Kyoto, Japan) and a UV Lambda 19 UV/VIS spectrophotometer (Perkin Elmer,
Waltham, MA), and were recorded on a computer connected to the spectrophotometers.

To compute the fourth derivative (4D) spectra, the software package Unscrambler (version 9.2, Camo, Oslo, Norway) was used. The 4D spectra by means of the Savitsky-Golay algortithm using a fourth polynomial fit over 25 points were chosen. The amplitudes of the signature peaks and troughs were obtained directly from the derivative spectra. Each sample was analyzed in duplicate on each machine.

When determining a continuous variable such as total protein using a wet chemical laboratory reference method, it is important to estimate the standard error of the laboratory reference method $\left(\mathbf{S}_{\text {ref }}\right)$. The $S_{\text {ref }}$ is an estimate of the precision of the reference method (Naes et al., 2002). The accuracy of the UV method is expressed either as root mean square error of prediction, root mean square error of cross validation, or standard error of performance. These prediction errors are defined in Naes et al. (2002). Another common measure of the quality of a calibration is the validated correlation coefficient between the predicted protein value, $\hat{y}_{i}$, and the measured reference protein value, $y_{i}$, also defined in Naes et al. (2002).

Regarding the results, an overview of the distribution of the different constituents of the 82 goat milk samples is presented in Table 1. The error of the laboratory/ reference method $\left(\mathrm{S}_{\text {Ref }}\right)$ was $0.028 \%$ total protein. For a new sample measured with the reference method as described above, the precision will, with $95 \%$ confidence, be expected to be $\pm 0.056 \%$ protein $\left(2 \mathrm{~S}_{\text {ref }}\right)$. The UV prediction results for determination of total protein in goat milk are presented in Tables 2 and 3 . The prediction results are similar both when comparing the 2 instruments (no significant difference) and when comparing the 3 data analysis methods. However, partial least squares (PLS) on 4D data gave a slightly lower prediction error for total protein $(P<0.05$, chi-squared test) compared with the 2 other methods. To illustrate

Table 2. Ultraviolet full cross-validated partial least square (PLS) and univariate linear regression (LR) prediction results for total protein (wt/ wt \%) of the 82 goat milk samples, measured on 2 instruments ${ }^{1}$

\begin{tabular}{|c|c|c|c|c|c|c|}
\hline Instrument $^{2}$ & $\begin{array}{l}\text { Regression } \\
\text { method }\end{array}$ & Transformation & $\begin{array}{l}\text { Wavelength } \\
(\mathrm{nm})\end{array}$ & $\mathrm{r}$ & RMSECV & $\begin{array}{l}\text { No. of } \\
\text { PLS }\end{array}$ \\
\hline \multirow[t]{2}{*}{ UV Lambda 19 UV/VIS spectrophotometer } & PLS & Abs & All & 0.93 & 0.12 & 5 \\
\hline & LR & $4 \mathrm{D}$ & 283.5 & 0.93 & 0.12 & - \\
\hline \multirow[t]{3}{*}{ UV-1601 visible spectrophotometer } & PLS & Abs & All & 0.93 & 0.12 & 5 \\
\hline & PLS & $4 \mathrm{D}$ & All & 0.93 & 0.12 & 1 \\
\hline & LR & $4 \mathrm{D}$ & 283.2 & 0.91 & 0.13 & - \\
\hline
\end{tabular}

${ }^{1}$ The correlation coefficients between the reference method and the UV predicted ( $\mathrm{r}$ ), the accuracy expressed as root mean square error of cross validation (RMSECV), and the number of PLS factors are presented. The regressions were performed on the raw absorbance (Abs) and on fourth derivative (4D) absorbance spectra. Bias was less than $0.01 \%$.

${ }^{2}$ UV Lambda 19 UV/VIS spectrophotometer: Perkin Elmer (Waltham, MA); UV-1601 visible spectrophotometer: Shimadzu (Kyoto, Japan). 


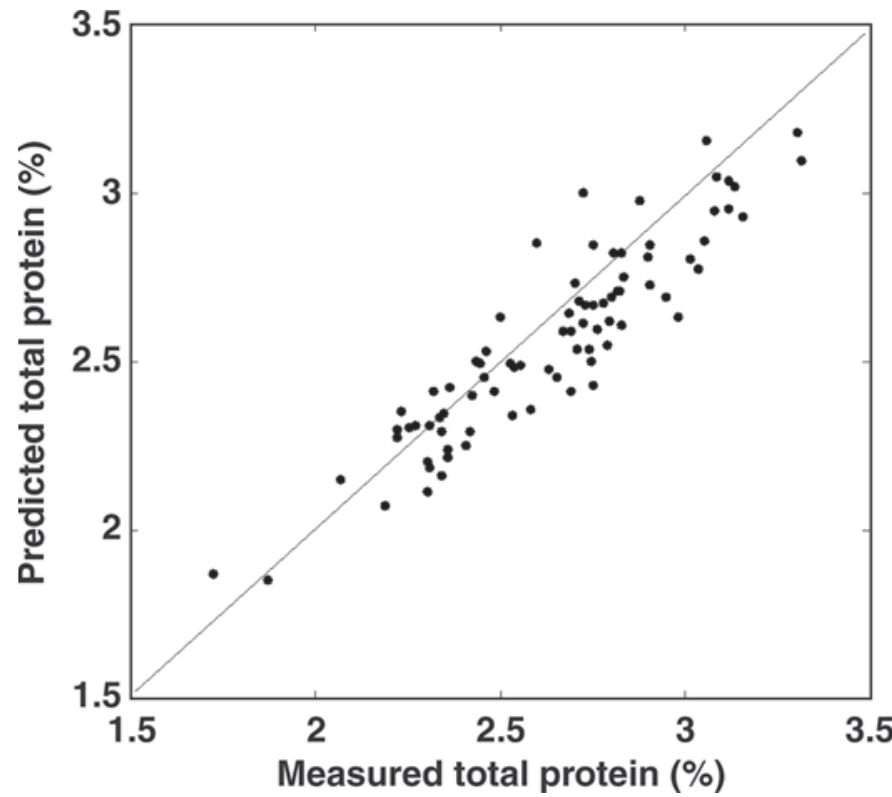

Figure 1. Measured total protein in goat milk plotted against predicted total protein for all samples $(\mathrm{n}=82)$; whole spectra, no preprocessing, using partial least squares, full cross validation, and 5 partial least squares factors (UV-1601 visible spectrophotometer, Shimadzu, Kyoto, Japan).

this accuracy, the UV predicted total protein versus reference method total protein is presented in Figure 1. The prediction errors can be interpreted in the following way: when measuring a new sample with the UV method, the reference method value is expected, with $95 \%$ confidence, to be within the UV predicted value $\pm 0.20 \%$ total protein. This loss of precision and accuracy, from \pm 0.056 to 0.20 , from the reference method to the UV method is the consequence of going from a laborious chemistry Kjeldahl method to a much faster spectroscopy method.

The calibration transfer prediction results (Table 3) clearly show that the prediction errors are substantially larger when using univariate regression on 4D spectra compared with multivariate regression (i.e., PLS). A reason for these variations is that a small (about $0.3 \mathrm{~nm}$ ) wavelengths shift was observed between the 2 studied instruments. Our recommendation based on the present results is to use multivariate regression (i.e., PLS) based on all available UV wavelengths instead of using $4 \mathrm{D}$ and univariate regression suggested by Lüthi-Peng and Puhan (1999). The conclusions from the results are as follows.

- The accuracy of determination or prediction of total protein in goat milk using UV spectroscopy gave similar results using either raw absorbance spectra or 4D spectra.

- Using 4D spectra, all wavelengths and multivariate PLS or a selected wavelength and univariate regression gave similar prediction results of total protein in goat milk.

- For calibration transfer (using a calibration model made on 1 instrument to predict components from spectra recorded on another instrument), multivariate PLS regression of whole spectra performs better than the use of univariate regression on $4 \mathrm{D}$ spectra.

- Multivariate (i.e., PLS) regression on raw absorbance UV spectra gave good accuracy for total protein in real goat milk using the chemical method described by Lüthi-Peng and Puhan (1999).

- Using selected peaks in spectra for determination of total protein in milk reported by Lüthi-Peng and Puhan (1999) did not give identical results on the 2 tested UV instruments in this study. This indicates a shift between the instruments. The described method by Lüthi-Peng and Puhan (1999) using $4 \mathrm{D}$ of selected peaks of spectra is therefore less robust regarding transferring measurements between UV instruments compared with this method using full spectra.

Table 3. Calibration transfer partial least square (PLS) and univariate linear regression (LR) prediction results for total protein (wt/wt \%) of the 82 goat milk samples, measured on 2 instruments ${ }^{1}$

\begin{tabular}{|c|c|c|c|c|c|c|c|c|}
\hline \multicolumn{2}{|c|}{ Instrument $^{2}$} & $\begin{array}{l}\text { Regression } \\
\text { method }\end{array}$ & Transformation & $\begin{array}{l}\text { Wavelength } \\
(\mathrm{nm})\end{array}$ & RMSEP & SEP & Bias & $\begin{array}{l}\text { No. of } \\
\text { PLS }\end{array}$ \\
\hline Cal & Test & PLS & $4 \mathrm{D}$ & All & 0.25 & 0.12 & 0.29 & 1 \\
\hline Cal & Test & LR & $4 \mathrm{D}$ & 283.2 & 0.22 & 0.11 & 0.19 & - \\
\hline Test & Cal & PLS & Abs & All & 0.14 & 0.13 & 0.07 & 3 \\
\hline Test & Cal & PLS & $4 \mathrm{D}$ & All & 0.16 & 0.12 & -0.12 & 1 \\
\hline
\end{tabular}

${ }^{1}$ The accuracy expressed as root mean square error of prediction (RMSEP) and as the bias-corrected standard error of performance (SEP) and the number of PLS factors are presented. The regressions were performed on the raw absorbance (Abs) and on fourth derivative (4D) absorbance spectra.

${ }^{2}$ Perkin Elmer = UV Lambda 19 UV/VIS spectrophotometer (Perkin Elmer, Waltham, MA); Shimadzu = UV-1601 visible spectrophotometer (Shimadzu, Kyoto, Japan). Cal and Test refer to calibration and test sets, respectively. 


\section{ACKNOWLEDGMENTS}

The authors thank the staff at the goat barn at The University of Life Sciences, the Department of Chemistry, Biotechnology and Food Science (Ås, Norway) and TINE Norwegian Dairies Association (Oslo, Norway) for technical assistance.

\section{REFERENCES}

Aschaffenburg, R., and J. Drewry. 1959. New procedure for the routine determination of the various non-casein proteins of milk. Pages 1630-1637 in Proc. XV International Dairy Congress. Vol. 3. Richard Clay and Co. Ltd., Bungay, Suffolk, UK.

Barbano, D. M., and J. M. Lynch. 1992. Crude and protein nitrogen bases for protein measurement and their impact on current testing accuracy. J. Dairy Sci. 75:3210-3217.

Devold, T. G., M. G. Brovold, T. Langsrud, and G. E. Vegarud. 2000. Size of native heated casein micelles, content of protein and minerals in milk from Norwegian Red Cattle-Effect of milk protein polymorphism and different feeding regimens. Int. Dairy J. $10: 313-323$.

IDF. 1962. International Standard 20. Determination of nitrogen content in milk-Part 1: Kjeldahl method. International Dairy Federation, Brussels, Belgium.
IDF. 1964. International Standards 29. Determination of casein content in milk. International Dairy Federation, Brussels, Belgium.

Liland, K. H., B. H. Mevik, E. O. Rukke, T. Almøy, and T. Isaksson. 2009. Quantitative whole spectrum analysis with MALDI-TOF MS, part II: Determining the concentration of milk in mixtures. Chemom. Intell. Lab. Syst. 99:39-48.

Lüthi-Peng, Q., and Z. Puhan. 1999. Determination of protein and casein in milk by fourth derivative UV spectrophotometry. Anal. Chim. Acta 393:227-234.

Miralles, B., B. Bartolomé, L. Amigo, and M. Ramos. 2000b. Comparison of three methods to determine whey protein to total protein ratio in milk. J. Dairy Sci. 83:2759-2765.

Miralles, B., B. Bartolomé, R. Ramos, and L. Amigo. 2000a Determination of whey protein to total protein ratio in UHT milk using fourth derivative spectroscopy. Int. Dairy J. 10:191-197.

Naes, T., T. Isaksson, T. Fearn, and T. Davies. 2002. A User-Friendly Guide to Multivariate Calibration and Classification. NIR Publications, West Sussex, UK.

Posati, L. P., and M. L. Orr. 1976. Composition of foods, dairy and egg products. Pages 77-109 in Agric. Handbook No. 8-1. USDA-ARS, Washington, DC.

Sherbon, J. W. 1978. Recent developments in determining protein content of dairy products by dye binding. J. Dairy Sci. 61:12741278 . 